\title{
Pancreatitis autoinmune: a propósito de un caso
}

\section{Autoimmune pancreatitis: a case report}

\author{
E. Valdivielso Cortázar'1, C. Prieto Martínez², I. Fernández-Urién², J.J. Vila Costas ${ }^{2}$, \\ F.J. Jiménez Pérez ${ }^{3}$, A. Guerra Lacunza ${ }^{2}$
}

http: //dx.doi.org/10.4321/S1137-6627/2016000100018

\section{RESUMEN}

La pancreatitis autoinmune (PAI) es una patología poco frecuente, aunque con una creciente incidencia en países orientales, si bien esto podría deberse a una mayor tasa de detección. Puede asociarse a otras patologías autoinmunes, y se ha descrito su asociación con la enfermedad sistémica por IgG4. La clínica es variada y el tratamiento habitualmente es médico, mediante corticoides.

Se describe un caso clínico diagnosticado.

Palabras clave. Pancreatitis autoinmune. IgG4.

\begin{abstract}
Autoimmune pancreatitis (AIP) is an infrequent pathology, although there is a growing incidence in Eastern countries, which might be due to a greater rate of detection. It can be associated with other autoimmune pathologies and its association with IgG4-related systemic disease has been described. Its clinical presentation is varied and the normal treatment is medical, using corticoids.

A diagnosed clinical case is described.
\end{abstract}

Keywords. Autoimmune pancreatitis. IgG4.
1. Servicio de Aparato Digestivo. Complejo Hospitalario Universitario de A Coruña.

2. Servicio de Aparato Digestivo. Complejo Hospitalario de Navarra. Pamplona.

3. Servicio de Aparato Digestivo. Hospital de la Ribera. Alzira. Valencia.

Recepción: 29-9-2015

Aceptación provisional: 28-10-2015

Aceptación definitiva: 4-11-2015

\section{Correspondencia:}

Eduardo Valdivielso Cortázar

Servicio de Aparato Digestivo

Complejo Hospitalario Universitario

Calle As Xubias, 84

15006 A Coruña

E-mail: eduardovaldi@hotmail.com 


\section{INTRODUCCIÓN}

La pancreatitis autoinmune (PAI) es una patología infrecuente, con mayor y creciente incidencia en países orientales, si bien esto podría deberse a una mayor tasa de detección ${ }^{1}$. Puede asociarse a otras patologías autoinmunes, y se ha descrito su asociación con la enfermedad sistémica por IgG4. La clínica es variada y el tratamiento habitualmente es médico, mediante corticoides $^{2}$.

\section{CASO CLÍNICO}

Presentamos el caso de un paciente de 44 años sin antecedentes de interés que ingresa en el servicio de Digestivo por cuadro de epigastralgia de 20 días de evolución, con coluria y acolia, además de sensación distérmica e hiporexia. El paciente no bebía alcohol. Analíticamente presentaba hiperbilirrubinemia (Bi: 4,5 mg/dl - VN: 0'2-1'2 mg/dl) de predominio directo, así como elevación de transaminasas (AST: $238 \mathrm{mg} / \mathrm{dl}-$ VN: 5-34 mg/dl; ALT: $609 \mathrm{mg} / \mathrm{dl}$ - VN: 5-55 mg/ dl ). Los triglicéridos y el calcio se encontraban dentro de los límites de la normalidad. Se realizó una ecografía que mostraba dilatación de la vía biliar con probable ocupación del colédoco por barro biliar. Se decidió realizar una CPRE (Fig.1) que mostraba un colédoco filiforme, de paredes irregulares y poco distensibles, con dilatación retrógrada, y sin barro en su interior, decidiendo tomar muestras para citología (negativa) y colocando una prótesis biliar plástica.

A los dos meses acudió a revisión en consultas, realizándose una analítica en la que se apreciaba normalidad en cuanto a los ANA, ANCA y las subclases de IgG. También de manera ambulatoria se realizó una colangio-RM sin hallazgos significativos, así como una ecoendoscopia que mostraba un engrosamiento difuso de la vía biliar y adenopatías en hilio hepático de hasta 4 $\mathrm{cm}$, realizándose PAAF de las mismas con citología negativa. Se retiró la prótesis plástica durante el mismo procedimiento. Se completó el estudio con un TC que mostraba vía biliar no dilatada, pero con engrosamiento de la pared, así como adenopatías de aspecto reactivo en hilio hepático.

Tres meses más tarde se realizó una ecografía de control que mostraba un área focal hipoecoica pancreática sin clara morfología de masa, así como una adenopatía de $1 \mathrm{~cm}$ de diámetro y un colédoco de $6 \mathrm{~mm}$ de calibre con pared engrosada. Se realizó una nueva ecoendoscopia con hallazgos similares a la realizada previamente, completando la exploración mediante una colangioscopia que mostraba una mucosa de la vía biliar de aspecto normal, si bien el conducto era menos distensible de lo normal. El paciente

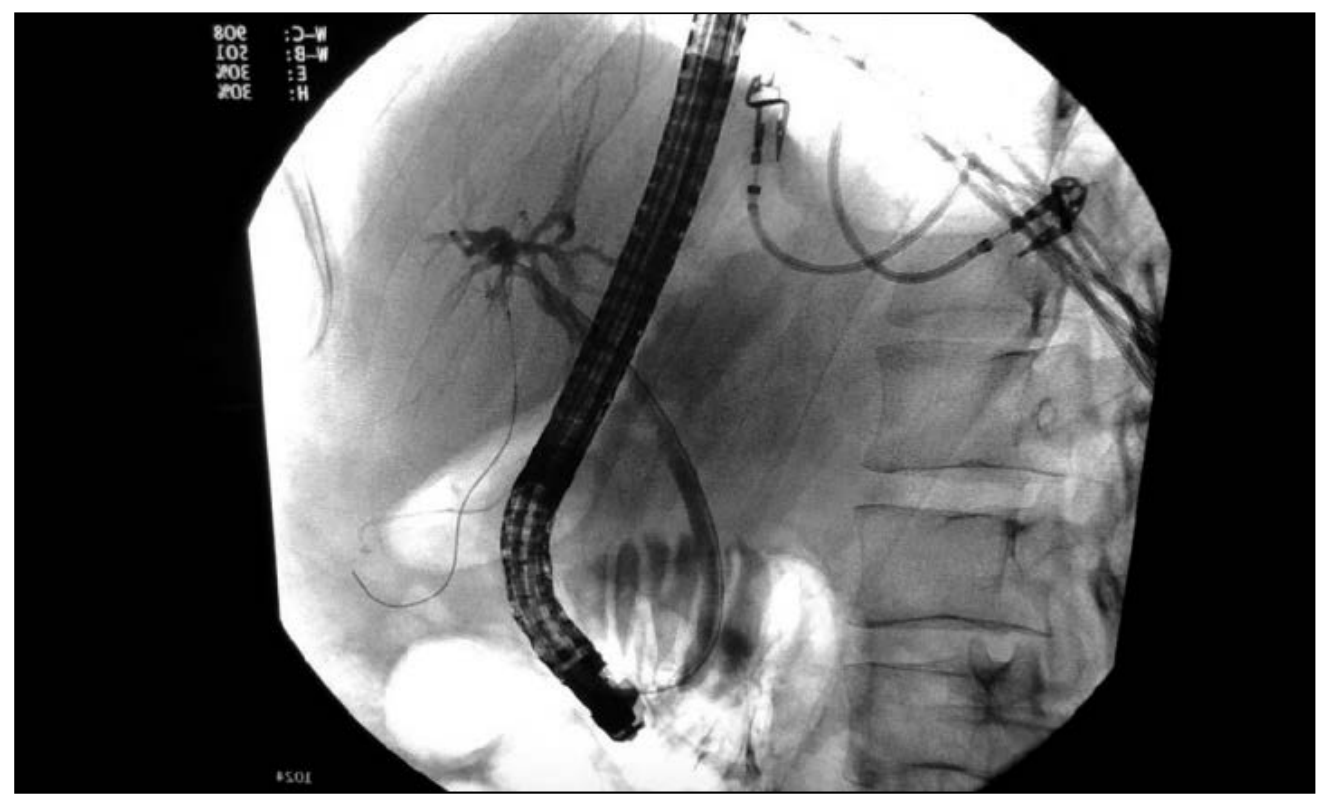

Figura 1. CPRE: estenosis de la vía biliar principal. 
refería encontrarse bien, aunque presentando algunos episodios de epigastralgia y distensión abdominal.

A los seis meses se repitió la ecoendoscopia apreciando un parénquima pancreático heterogéneo, con halo hipoecoico alrededor del Wirsung, que presentaba refuerzo ecogénico de la pared. En la cola de páncreas se apreciaba una masa hipoecoica de $3 \mathrm{~cm}$ de diámetro, heterogénea y bien delimitada, con centro hipoecoico y disposición radial, que no parecía afectar a órganos vecinos. Se puncionó con aguja de $25 \mathrm{G}$ remitiendo el material para estudio citológico, apreciándose infiltración linfoplasmocitaria y estroma celular inflamatorio, compatibles con pancreatitis autoinmune, pero con inmunorreactividad negativa para IgG4. El colédoco presentaba además un engrosamiento difuso hipoecoico de la pared con calibre y contenido normal. Todo ello era sugestivo de pancreatitis autoinmune, de modo que se inició tratamiento corticoideo en pauta descendente con buena evolución clínica y radiológica a los dos meses.

\section{DISCUSIÓN}

La pancreatitis autoinmune (PAI) es una patología infrecuente ${ }^{1}$ que puede dar manifestaciones a distintos niveles. A nivel pancreático se pueden apreciar masas pancreáticas ${ }^{3}$; dolor abdominal leve con o sin episodios de pancreatitis aguda ${ }^{4}$; o estenosis del conducto pancreático principal ${ }^{5}$. La PAI suele manifestarse como ictericia obstructiva, debido al desarrollo de estenosis biliares, que suelen asociarse con mayor frecuencia a una elevación de los niveles de IgG4 ${ }^{6}$. Además, también pueden aparecer nódulos pulmonares, síndrome de Sjögren asociado, tiroiditis autoinmune o nefritis intersticial ${ }^{7}$.

Existen dos tipos de PAI: el tipo 1 se asocia a la enfermedad sistémica por IgG4 y a una mayor incidencia de recidivas ${ }^{8}$; el tipo 2, en cambio, se caracteriza por una pancreatitis ducto-céntrica idiopática, en ocasiones con granulomas, pero sin inmunorreactividad a IgG4, con menos tendencia a recurrir, menor afectación sistémica, pero con una mayor asociación a la enfermedad inflamatoria intestinal, sobre todo a la colitis ulcerosa. La prevalencia de todos los subtipos de PAI es baja, si bien la PAI tipo 2 es muy poco frecuente en Japón, re- presentando menos del $10 \%$ de los casos de PAI; en cambio, en Europa y EEUU la PAI tipo 2 llega a representar el 40-60\% de los casos. El tabaquismo parece asociarse a una mayor frecuencia de diabetes y cambios morfológicos persistentes pancreáticos en pacientes con PAI, así como a una mayor tendencia a presentar recidivas e insuficiencia pancreática exocrina ${ }^{9}$. Además, se ha visto que el desarrollo de insuficiencia pancreática endocrina y exocrina es frecuente en estos pacientes, siendo necesaria una evaluación periódica de las $\operatorname{mismas}^{10}$.

En 2010, la Asociación Internacional de Pancreatología elaboró el Consenso Internacional sobre Criterios Diagnósticos ${ }^{11}$. Para el diagnóstico de la PAI, se deben estudiar 5 aspectos (Tabla 1), que se categorizan en dos niveles según su fiabilidad diagnóstica. Tras aplicar estos criterios, podremos decir si hay un diagnóstico definitivo o probable de PAI tipo 1 o 2, aunque en ocasiones no es posible realizar esta distinción (PAI indeterminada). En el caso de la PAI tipo 2, es necesaria la confirmación histológica para poder llegar a un diagnóstico definitivo.

El tratamiento se basa en el empleo de corticoides, sin que exista de momento un protocolo estandarizado de tratamiento. No obstante, puede comenzarse con 35-40 $\mathrm{mg} /$ día de prednisona durante 4 semanas y, si hay respuesta clínico-radiológica, disminuir gradualmente la dosis durante 3-4 meses. Algunos grupos recomiendan el mantenimiento a dosis bajas en la PAI tipo 1 debido a su mayor tasa de recurrencia, pudiéndose plantear también el tratamiento con azatioprina, metotrexato o micofenolato.

En nuestro caso llama la atención la presencia de afectación de la vía biliar sin que haya elevación de IgG4. Aunque el paciente no cumple todos los criterios diagnósticos, los hallazgos radiológicos junto con la buena respuesta clínico-radiológica al tratamiento corticoideo hacen pensar en una pancreatitis autoinmune tipo 2. Dado que existe la posibilidad de recidivas en estos tipos de pancreatitis ${ }^{12}$, se aconseja el seguimiento clínico de estos pacientes. 
Tabla 1. Criterios diagnósticos propuestos por el CICD (2010)

\begin{tabular}{|c|c|c|c|}
\hline & Criterio & Nivel 1 & Nivel 2 \\
\hline $\mathbf{P}$ & Imagen del parénquima & Aumento difuso con realce retardado & $\begin{array}{l}\text { Aumento focal con realce } \\
\text { retardado }\end{array}$ \\
\hline D & Imagen ductal & $\begin{array}{l}\text { Estenosis Wirsung }>1 / 3 \text {, o múltiples, } \\
\text { sin dilatación distal marcada }\end{array}$ & $\begin{array}{l}\text { Estenosis segmentaria/focal } \\
\text { sin dilatación ductal marcada } \\
\text { (Wirsung }<5 \mathrm{~mm} \text { ) }\end{array}$ \\
\hline AOO & Afectación de otros órganos & - & $\begin{array}{l}\text { Enfermedad inflamatoria } \\
\text { intestinal }\end{array}$ \\
\hline $\mathbf{H}$ & Histología & $\begin{array}{l}\text { Pancreatitis ductocéntrica idiopática: } \\
\text { - Infiltración granulocítica de pared } \\
\text { ductal con o sin inflamación acinar } \\
\text { granulocítica. } \\
\text { - No inmunorreactividad para IgG4. }\end{array}$ & $\begin{array}{l}\text { - Infiltración acinar } \\
\text { granulocítica y } \\
\text { linfoplasmocitaria. } \\
\text { - No inmunorreactividad para } \\
\text { IgG4. }\end{array}$ \\
\hline Rt & Respuesta a esteroides & \multicolumn{2}{|c|}{$\begin{array}{l}\text { Resolución radiológica en menos de } 2 \text { semanas o marcada mejoría de } \\
\text { las manifestaciones pancreáticas y/o extrapancreáticas. }\end{array}$} \\
\hline
\end{tabular}

\section{BIBLIOGRAFÍA}

1. OкаZакі K. Autoinmune pancreatitis is increasing in Japan. Gastroenterology 2003; 125: 1557-1558.

2. Kamisawa T, Egawa N, Nakajima H. Autoinmune pancreatitis is a systemic autoimmune disease. Am J Gastroenterol 2003; 98: 2811-2812.

3. Raina A, Yadav D, Krasinskas AM, McGrath KM, KhAlid A, SANDERS M et al. Evaluation and management of autoimmune pancreatitis: experience at a large US center. Am J Gastroenterol 2009; 104: 2295-2306.

4. Frulloni L, Scattolin C, Falconi M, Zamboni G, CAPElLi P, MANFredi R et al. Autoimmune pancreatitis: differences between the focal and diffuse forms in 87 patients. Am J Gastroenterol 2009; 104: 2288-2294.

5. SAhani DV, KalVA SP, FarRell J, Maher MM, SAINI S, MUELLER PR et al. Autoimmune pancreatitis: imaging features. Radiology 2004; 233: 245352.

6. Sandanayake NS, Church NI, Chapman MH, JohNSon GJ, Dhar DK, Amin Z et al. Presentation and management of post-treatment relapse in autoimmune pancreatitis/immunoglobulin G4-associated cholangitis. Clin Gastroenterol Hepatol 2009; 7: 1089-1096.

7. Chari ST, Takahashi N, Levy MJ, Smyrk TC, Clain JE, Pearson RK et al. A diagnostic strategy to distinguish autoimmune pancreatitis from pancreatic cancer. Clin Gastroenterol Hepatol 2009; 7: 1097-1103.

8. Bujus J, Cahen DL, van Heerde MJ, Rauws EA, De BUy WenNiger LJ, HANSEn BE et al. Long-term relapse rates of type 1 and type 2 autoimmune pancreatitis. Gastroenterology 2014; 146 Suppl 1: S-804.

9. Maire F, Rebours V, Vullierme MP, Couvelard A, LÉvY P, HENTIC O et al. Does tobacco influence the natural history of autoimmune pancreatitis? Pancreatology 2014; 14: 284-288.

10. Buiss J, Cahen DL, van Heerde MJ, Rauws EA, de Buy Wenninger LJ, Hansen BE et al. The longterm impact of autoimmune pancreatitis on pancreatic function, quality of life and live expectancy. Pancreas 2015; 44: 1065-1071

11. Shimosegawa T, Chari ST, Frulloni L, Kamisawa T, Kawa S, Mino-Kenudson M et al. International Association of Pancreatology. International consensus diagnostic criteria for autoimmune pancreatitis : guidelines of the International Association of Pancreatology. Pancreas 2011; 40: 352-358.

12. Hart PA, Topazian MD, Witzig TE, Clain JE, GleESON FC, KLEBIG RR et al. Treatment of relapsing autoimmune pancreatitis with immunomodulators and rituximab: the Mayo Clinic experience Gut 2013; 62: 1607-1615. 